\title{
Multi-Gigabit Wireless Data Transfer for High Energy Physics Applications
}

\author{
H.K. Soltveit ${ }^{* \dagger}$ \\ Ruprecht-Karls-Universitaet Heidelberg (DE) \\ E-mail: Soltveitaphysi.uni-heidelberg.de
}

\begin{abstract}
The future of connectivity is wireless and in that context the High Energy Physics (HEP) community is no exception. The volume of data produced at the Large Hadron Collider (LHC) is immense and represents a considerable processing challenge. Moreover the LHC and future hadronic colliders are the most demanding environments, with respect to ultra-low latencies, ultra-high data rate, reliable indoor coverage and harsh radiation environment in the world.

Any technology meeting the above conditions will have to satisfy the standard requirements of energy efficiency, low cost and security. Today, the data transmission in HEP experiments is based on wire and optical readout architecture. We propose the use of wireless data transmission described here as a third option. This paper presents the motivation and overviews of the status of our ASIC development and first ideas for wireless readout and control. In this first phase of development, we will focus on the $60 \mathrm{GHz}$ band, which provides high bandwidth, small form factor and is a mature technology. Open issues and future strategy are also briefly discussed.
\end{abstract}

EPS-HEP 2017, European Physical Society conference on High Energy Physics 5-12 July 2017

Venice, Italy

${ }^{*}$ Speaker.

${ }^{\dagger}$ On behalf of the WADAPT Consortium. 


\section{Introduction}

The 4th industrial revolution is on the way, and in front of us is a new era of wireless data traffic/transfer approaching fast. Wireless communication is actually the fastest growing part in the communication industry at time and is actually all around us. The driver behind this revolution is the rapid transistor technology evolution, which triggered the opening of the mm-wave band, that in turn opens the doors for Internet of Things (IoT), smart homes, 5G, autonomous automotive industry, cloud computing and a myriade of opportunities in healthcare, etc. In all these aspects there is pressure towards higher and higher bandwidth, where "Edholms law" predicts that wireless data volumes would double every 18 months. It is anticipated that the development of these cuttingedge devices will totally change the way we live, work and interconnect with each other. This trend towards a wireless future will surely affect and drive the developments for future instrumentation in the HEP community.

The WADAPT ${ }^{1}$ consortium proposes wireless data transmission as an alternative to wire and optical readout architecture presently in use in HEP experiments. It is well known that, with almost all the available spectrum below $6 \mathrm{GHz}$ being allocated, it is mandatory to move to higher frequency bands to secure bandwidth. However, as the frequency increases, RF propagation and penetration through materials etc. are reduced, which will prevent wireless data leaking from one sector to adjacent sectors. Thus, the bandwidths available above $6 \mathrm{GHz}$ lend themselves to short-range, point-to-point, Line-Of-Sight (LOS) connections. The band that has attracted the highest interest in this context is the mm-wave band. The mm-wave band is defined where the wavelength varies from ten millimeters $(30 \mathrm{GHz})$ down to 1 millimeter $(300 \mathrm{GHz})$. In the first phase the WADAPT consortium [1] will concentrate on data transfer communication in the $60 \mathrm{GHz}$ band (57 GHz - 66 $\mathrm{GHz}$ ), where the $9 \mathrm{GHz}$ license free band is extremely attractive in order to achieve a high data rate transfer.

The major reasons for using wireless communication in a HEP like environment is due to all the advantages it brings in terms of cable (material) reduction, small form factor, high material penetration loss, high free path loss, narrow beamwidth, flexible deployment, easy maintenance, fiber comparable data rate transfer, ultra-low latency, high quality, reliability, security and implementation of new applications such as wireless control. All in all this will reduce the overall cost of the present detector readout system. In addition, the wireless communication technology is not constrained to mechanical structures, like for example optical links and wires that has to follow the structure of the given construction. All these features, added to the operation in a very well controlled environment with LOS operation, make the $60 \mathrm{GHz}$ band optimal for short range operation as in a closed detector environment.

The planning and construction of the existing detectors were done in the "Pre-Wireless age", and the future need of extreme data rates in the order of $10 \mathrm{~Gb} / \mathrm{s}$ and beyond in a compact and densely populated detector facility. Therefore, to fully exploit the advantages of integrating a fully wireless point-to-point readout, a re-thinking of the detector topology is mandatory. This means the coverage, the capacity demand and the topology for the specific detector. Understanding these aspects from the wireless point of view, will help constructing an optimum distribution strategy for all of the HEP requirements.

\footnotetext{
${ }^{1}$ Wireless Allowing Data and Power Transmission
} 
Wireless communication also leads to a more efficient system control management. A wireless control solution provides reliable, flexible and secure operation that will allow a greater control while reducing total implementation and maintenance cost. Higher performances and more reliable communications with respect to current technologies are expected from these characteristics.

Wireless data transmission could help to reduce the number of cables (optical or copper) used for data transmission as well as their connectors, which are prone to damage. With less cables and connectors, installation and repair of such a complex system like a tracking detector can be simplified. Moreover, cables contribute significantly to the material budget in the active detector volume, which leads to multiple scattering and acts as a source of secondary particles. For example a wireless control solution will make it possible to transfer large amount of data in both directions in real-time with minimum latency to a large number of individual devices; this would be impracticable by using wire or optical connections, which are bound to follow the mechanical architecture. This could be used to enhance on-detector intelligence such as on-line finding of tracks with high transverse momentum for a fast trigger as the data could be read along particle trajectories. To increase the efficiency of the detector an instantaneous optimization based on real-time monitoring of the performance of the sub-components is also possible. This could be interesting for detector synchronization or for the distribution of a system wide reset or trigger signal. Also increased processing capabilities will be needed to cope with increasing levels of intelligence required to process complex stimuli in real-time. The amount of data, control signal to share/use and to make on-the spot decision has a significant potential. For example Figure 1 shows several possible scenarios of new topologies that can be created more easily than using wire or optical links. '
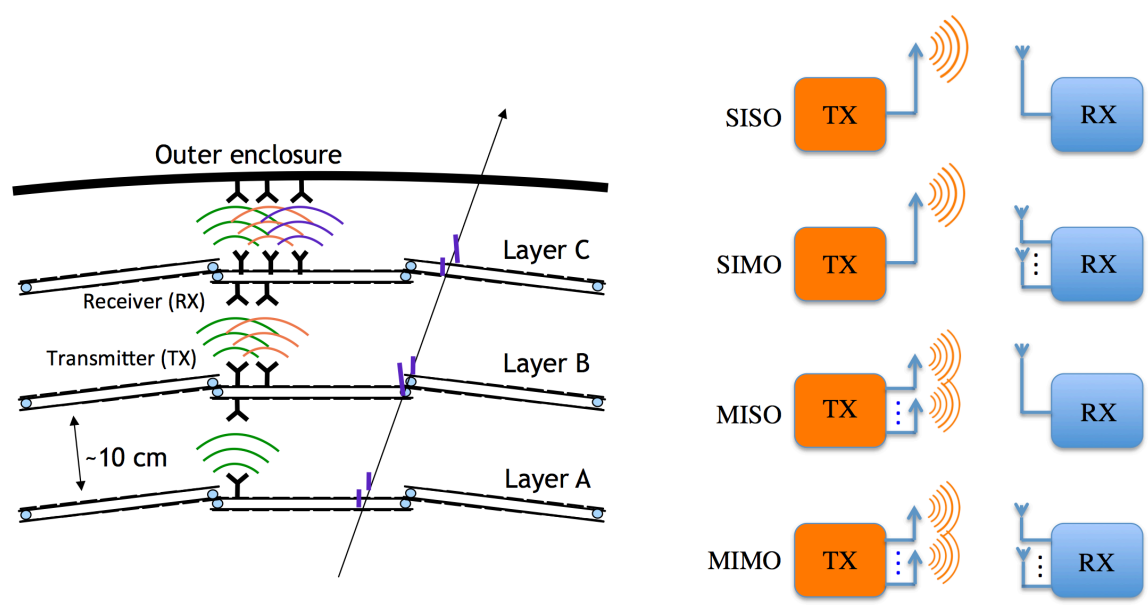

Figure 1: Left: Proposal of a radial readout for the tracker detector of the ATLAS experiment [2] . Right: Different readout/control compositions that can be created using a wireless realization. Single Output transmitter, Single Input receiver (SISO), Single Input receiver, Multiple Output transmitter (SIMO), Multiple Input receiver, Single Output transmitter (MISO), Multiple Input receiver, Mutiple Output transmitter (MIMO)

Not all of the sensors need to be operated in the mm-wave band, some may communicate at low-bit-rates but with ultra-low latency and ultra-high reliability, and may require reliable highbandwidth communication. An example could be transfer of a huge amount data for fast intervention, maintenance or assembly tasks, or functions such as remote control of other modules. 
In this development many requirements have to be fulfilled at similar or lower cost and energy dissipation as the existing system.

\section{Transceiver Design}

Several different wireless transceiver topologies and IC technologies exist, which are very specification dependent and will not be discussed in this paper. The first prototype of the Heidelberger transceiver chip as shown in Figure $2[3,4]$ is designed to handle a data rate of $4.5 \mathrm{Gbps}$ over a link distance of about $1 \mathrm{~m}$.

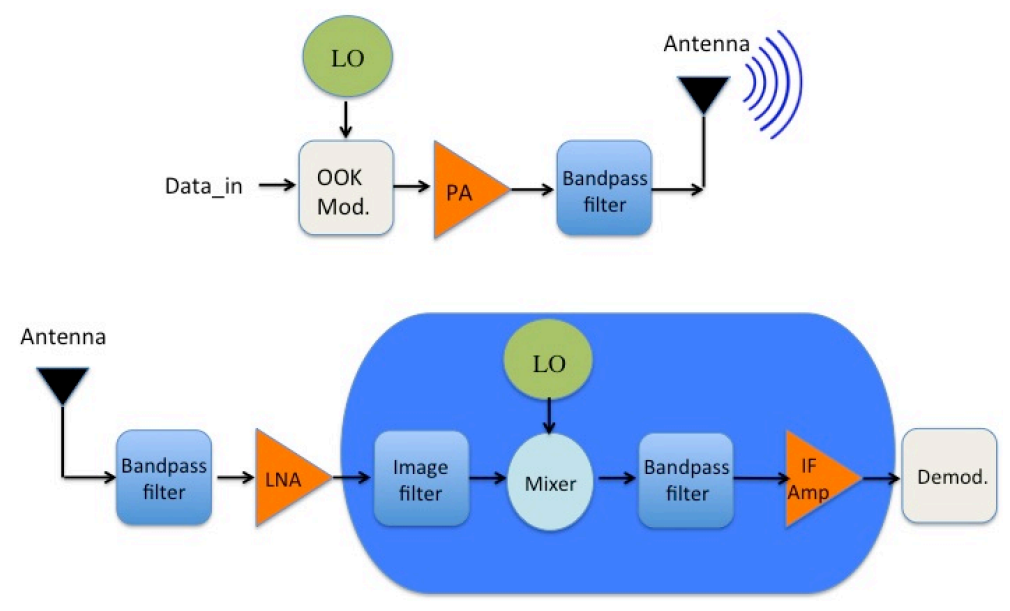

Figure 2: Block diagram of the Transceiver. The transmitter is shown at the top and the receiver at the bottom.

With the present design a total estimated power consumption for the first prototype is about 150 $\mathrm{mW}$. This first prototype design will be taped-out in IBM SiGe BiCMOS $130 \mathrm{~nm} 8 \mathrm{HP}$ technology. In the $60 \mathrm{GHz}$ band a bandwidth of $9 \mathrm{GHz}$ is available. This allows for high data rates and low complexity modulation schemes.

\begin{tabular}{|c|c|}
\hline Specifications & Value \\
\hline Frequency band & $57-66 \mathrm{GHz}$ \\
\hline Bandwidth & $\mathbf{9 ~ G H z}$ \\
\hline Data Rate & $4.5 \mathrm{Gbps}$ \\
\hline Modulation & $\mathrm{OOK}$ \\
\hline $\begin{array}{c}\text { Minimum sensitivity } \\
\mathrm{S}_{\mathrm{rx}(\min )}\end{array}$ & $-49 \mathrm{dBm}$ \\
\hline Bit Error Rate (BER) & $10^{-12}$ \\
\hline Target Power consumption & $150 \mathrm{~mW}$ \\
\hline Transmission Range & $20 \mathrm{~cm}(1 \mathrm{~m})$ \\
\hline
\end{tabular}

Figure 3: Summary of the specifications used for the ASIC design.

The power consumption is heavily dependent on specifications like data rate, transfer distance and antenna gain. There is room for improvements regarding power consumption and the effort will be pursued in that direction. The initiated transceiver chip development is foreseen to grow in complexity and functionality as we explore the possibilities. One ultimate scenario could be 
to integrate/readout the exciting ABCn chip of ATLAS with the transceiver chip implemented in same topology to create a System-on-Chip $(\mathrm{SoC})$ or using 3D integration as shown in Figure 7 This offers significant advantages when it comes to further minimizing the mass, volume, power consumption and development cost, while giving the possibility of increased data rate and channel density.

a

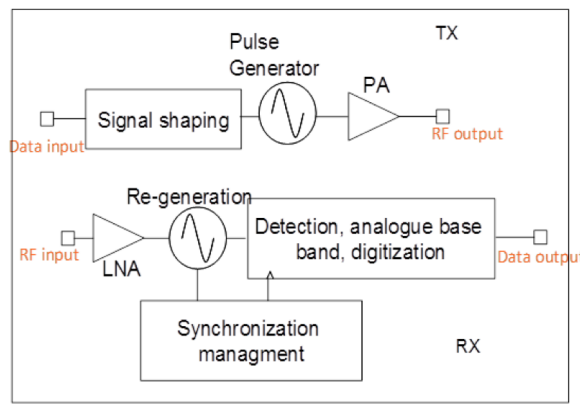

b

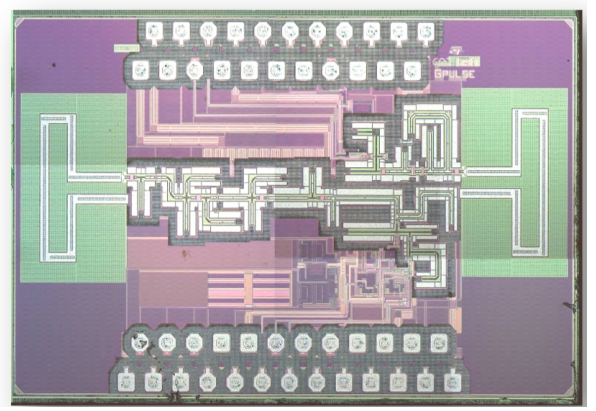

Figure 4: Left: Block diagram of the transceiver. The transmitter is shown at the top and the receiver at the bottom. Right: Layout of the CEA-Leti Chip

CEA-Leti has co-developed a transceiver with industrial partners as shown in Figure 4. The CEA-Leti $60 \mathrm{GHz}$ chip is able to reach $5 \mathrm{Gbps}$ and $10 \mathrm{Gbps}$ over a short distance (few centimeters) with a BER $<10^{-12}$ and a power consumption of $40 \mathrm{~mW}$ in transmission and $20 \mathrm{~mW}$ in reception [5]. The range is $0.2 \mathrm{~m}$ with single antenna with a scalable data rate from $100 \mathrm{Mbps}$ to 2.5 Gbps. This prototype undergoes now the first irradiation tests to investigate potential issues under operation.

\section{Antenna Design}

The antenna is a critical component in the transceiver chain. The $60 \mathrm{GHz}$ carrier frequencies and above enable antennae to be placed much closer together than $2.4 \mathrm{GHz}$ or $5 \mathrm{GHz}$ carrier frequencies. Antennae operating at such high frequency are typically very directional. In fact, multiple antennas can be placed on a single chip that can be used for beamforming to increase the received power. Operation in the $60 \mathrm{GHz}$ or mm-wave band also makes it possible the use of massive antenna configurations on-chip, which makes intelligent wireless control feasible.

The work of the Uppsala group ${ }^{2}$ encompasses the design and simulation of various antenna types for usage in detector environments, their fabrication and characterization. The first irradiation with electrons of $5 \mathrm{Mev}$ (100 krad dose), $120 \mathrm{Mev}$ (100 krad and $10 \mathrm{Mrad}$ ) have been conducted with very positive outcome [6].

\section{Feasibility Study}

To evaluate the influence and other potential issues that could appear under operation of the $60 \mathrm{GHz}$ RF signal on electronics and material used for the ATLAS tracker readout in this case, a

\footnotetext{
${ }^{2}$ University of Uppsala, Department of Physics and Astronomy
} 

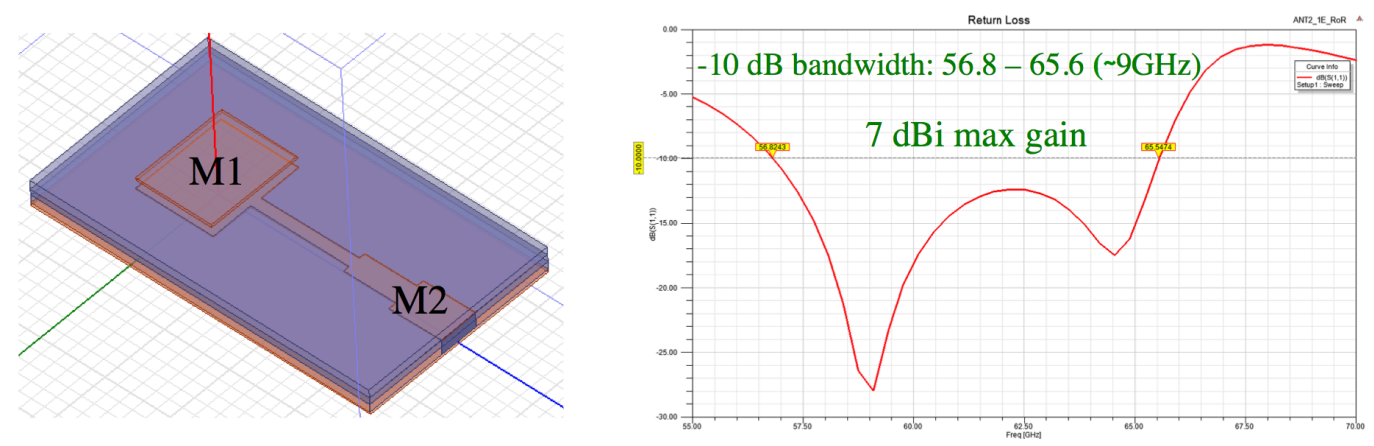

Figure 5: Left: Antenna design. Right: Simulated antenna response

$60 \mathrm{GHz}$ Evaluation Kit from Hittite was installed and the following measurements were completed in Heidelberg ${ }^{3}$ : data transmission, material properties, antenna characterization, cross-talk, link density and noise pick-up. A very important aspect is to understand how the circuit behaves and influences other circuits in the vicinity of the transceiver. No influence on transceiver chips working in the $60 \mathrm{GHz}$ band from other chips in the vicinity are expected. The many metallic surfaces could lead to strong multipath components of the radio signal that may cause fading. Tests with an ITK hybrid with $\mathrm{ABCn} 130 \mathrm{~nm}$ readout ASICs showed no observable effect on the module's noise performance. A detailed description of the above tests can be found in [7] .

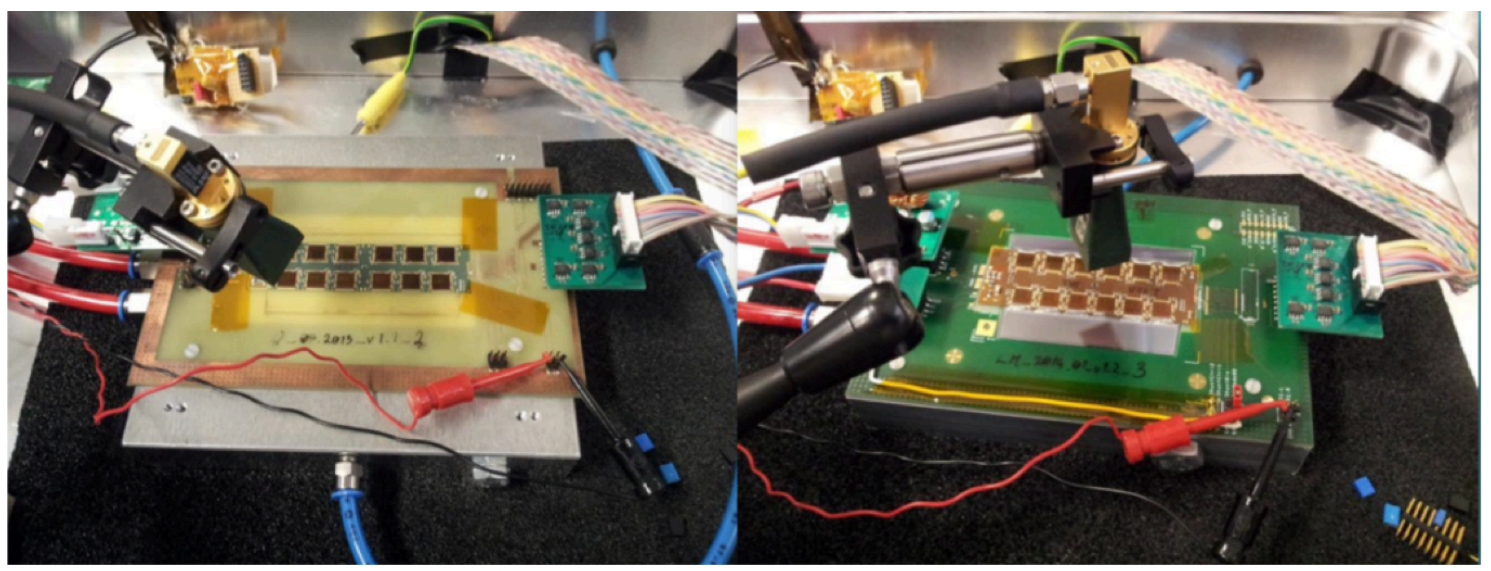

Figure 6: Experimental test setup used in Heidelberg for the $60 \mathrm{GHz}$ RF signal irradiation of the ABCn 130 $\mathrm{nm}$ readout ASICs .

\section{Future Strategy and open issues}

There are many possibilities but also some open issues. Since this wireless readout development is planned for the next generation of detector systems, a major focus in this development is naturally on the power consumption, space, reliability, and for some sub-systems it has to be extremely radiation hard. For example the wireless transceiver ASIC will be exposed to about

\footnotetext{
${ }^{3}$ Physikalisches Institut, University of Heidelberg
} 
10 times higher radiation dose than the current LHC ASICs in the worst case. Thus a deep understandig of the final system application and applied IC technology is mandatory. Significant technological evolution can also be expected in the coming years. What technology to use and where to use it in the detector depends on many factors. For example for which application is IC radiation hard technology needed, what are the bandwidth requirements for the different modules, where is analog performance and feature size relevant, where is power consumption important.
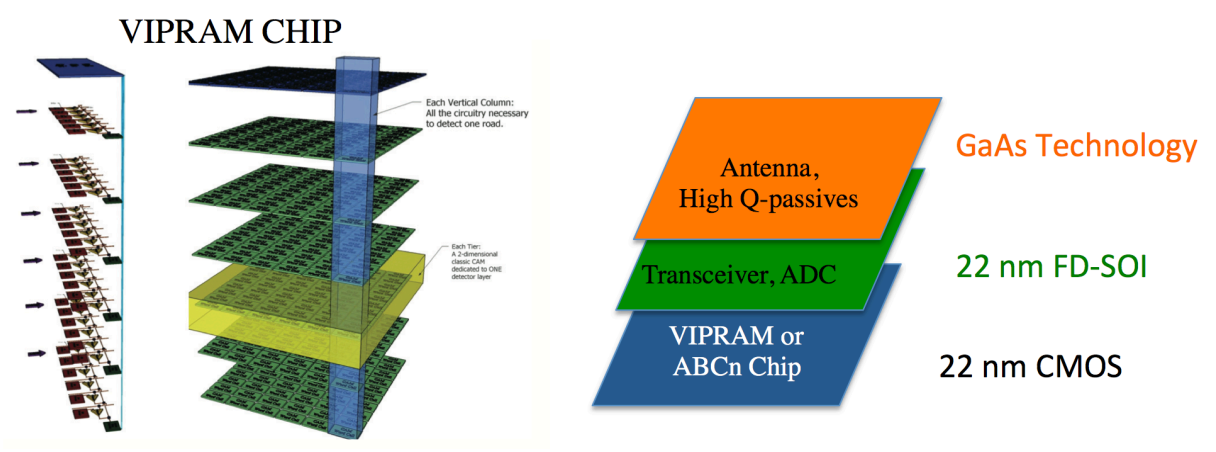

Figure 7: Left: Topology of the VIPRAM chip [8]. Right: Possible stacking strategy including technlogy, where the optimal choice of technology or the combination of technologies will depend on the specific user case.

There are applications in the detector environment that could potentially gain from the ultralow latency. With the possibility of ultra-low latency applications, a latency budget analysis of the current/future data transmission path will be a first step towards reduced latency and finding new applications. The main question is where this ultra-low latency offers new opportunities in the form of applications or services. For this, the focuses have to be super real-time and reliable connections, new applications and cases where very strict requirements on latency and reliability are needed. Moreover, the way to wirelessly handle/control a large number of devices, and to handle a large number of links in an extremely dense environment as a detector environment has to be defined.

The next big challenge is to evaluate the benefit for the HEP community, which could result from a fully or partial designed wireless power distribution in/off detector when it comes to the enormous potential of cable reduction.

Since the future detectors will be highly granular, and maybe un-triggered, a large amount of data has to be readout in short time, and will require high bandwidth and compact design to read out the data in Terabits per second. An attractive solution is therefore to integrate the wireless transmitter onto the already existing readout chip, for example the VIPRAM chip [8] or the existing $\mathrm{ABCn}$ chip for ATLAS, which then will be the first chip of this kind in the world. With this solution, a further improvement in form factor, power consumption, functionality etc. is foreseen.

\section{Summary}

This paper describes the first steps of the WADAPT consortium in this innovative development with potentially huge outreach, by providing a practical alternative to wire and optical links 
to readout large amount of data. Each of the three options has pros and cons and is extremely application dependent. A close collaboration with the HEP community to evaluate these three options for optimum usage is needed.

As already mentioned above the environment of the LHC and future colliders is one of the most demanding in the world for wireless data processing, with respect to ultra-low latencies, cross-talk, ultra high data rate, reliable indoor coverage, harsh radiation environment etc., on top of the standard detector requirements of energy efficiency and low cost. The wireless solution has many advantages compared to existing readout systems. Among them are cable/material reduction, small form factor, controllable beamwidth and data transfer intensity (length). In addition high path loss, high penetration loss leads to the absence of cross-talk between adjacent modules and make the frequency reuse possible. The flexible deployment, easy maintenance, ultra-low latency, fiber like data rate transfer, high quality, high reliability, security, the absence of constrain to mechanical structures, contrarily to wire and optical links, make it easier the implementation of new applications like wireless control. Moreover wireless data processing is an extremely challenging opportunity with unprecedented potential for commercial exploitation.

New readout topologies are possible which have the potential of providing a more efficient system control that can minimize downtime, and provide feedback control to enhance system quality. It is well known that the optical link connection has the highest peak data transfer rate possible today due to its enormous bandwidth. Wireless is free of wires going everywhere, it can communicate to multiple devices where wire or optical link is costly or infeasible to install. The optimum solution is highly case dependent. The mmWave band offers the possibility of a new generation of readout with very low latencies and massive bandwidth. The wireless readout needs to match the existing network in terms of delivered quality, reliability and security. To do so, we need in the long term to deliver fiber like $10 \mathrm{~Gb} / \mathrm{s}$ speed.

The future of connectivity is wireless and the HEP community will surely draw great benefit from this developing technology. The success of this project can in addition enhance the global competitiveness and the cutting edge wireless technology.

\section{References}

[1] E. Locci et al., Development of wireless techniques in data and power transmission - application for particle physics detectors, 1511.05807.

[2] R. Brenner and S. Cheng, Multigigabit wireless transfer of trigger data through millimeter wave technology, in Workshop on Intelligent Trackers 2010.

[3] H. K. Soltveit, R. Brenner, A. Schoning and D. Wiedner, Multi-Gigabit Wireless data transfer at 60 GHz, JINST 7 (2012) C12016, [1206.2287].

[4] H. K. Soltveit, S. Dittmeier, A. Schoning and D. Wiedner, Towards Multi-Gigabit readout at $60 \mathrm{GHz}$ for the ATLAS silicon microstrip detector, Nuclear Scienece Symposium and Medical Imaging Conference (NSS/MIC), 2013 IEEE (2013) 1-6.

[5] J. Luna and et al., A packaged $60 \mathrm{GHz}$ low power transceiver with integrated antenea for short range communication, Radio and Wireless symposium (RWS) 2013 IEEE (2013) 355.

[6] D. Pelikan, N. Bingefors, R. Brenner, D. Dancila and L. Gustafsson, Wireless data transfer with mm-waves for future tracking detectors, Journal of Instrumentation 9 (2014) C11008. 
[7] Dittmeier, Sebastian and Schoning, Andre and Soltveit, Hans Kristian and Wiedner, Dirk , Feasibility studies for a wireless $60 \mathrm{GHz}$ tracking detector readout, Nucl. Instrum. Meth. A830 (2016) 417-426, [1604.06259].

[8] T. Liu, G. Deptuch, J. Hoff, S. Jindariani, S. Joshi, J. Olsen et al., Design and testing of the first $2 d$ prototype vertically integrated pattern recognition associative memory, Journal of Instrumentation 10 (2015) C02029. 